\title{
Die Behandlung der Condylus-radialis-humeri-Fraktur im Kindesalter
}

\author{
Nawid Ayubi, Johannes M. Mayr, Sergio Sesia, Rainer Kubiak
}

\section{Zusammenfassung \\ Operationsziel}

Operative Reposition und Retention des Condylus radialis humeri zur Vermeidung von bleibenden Fehlstellungen, Pseudarthrosen und Gelenkinstabilitäten.

\section{Indikationen}

Absolut: Primär vollständig dislozierte Frakturen oder Frakturen, die nach 4 Tagen im gipsverbandfreien Kontrollröntgen eine Sekundärdislokation von > 2 mm aufweisen.

Relativ: Komplette Frakturen des Condylus radialis humeri, die im Verlauf eine Sekundärdislokation von $\leq 2 \mathrm{~mm}$ aufweisen.

\section{Kontraindikationen}

Inkomplette, sog. hängende Frakturen des Condylus radialis humeri ohne erkennbare Sekundärdislokation im Verlauf.

\section{Operationstechnik}

Offene Reposition des Condylus radialis humeri über einen lateralen Zugang am Ellenbogengelenk. Bei jüngeren Kindern ( $<5$ Jahre) Kirschner-Draht-Fixierung. Bei älteren Kindern ( $\geq 5$ Jahre) oder bei unzureichender Kompression Durchführung einer radialen Zugschraubenosteosynthese. Zusätzliche Periostnaht.

\section{Weiterbehandlung}

Anlage einer Oberarmgipslonguette bis zum Abschluss der Wundheilung; im Anschluss Oberarmgipsverband für insgesamt 3-4 Wochen nach der Operation. Materialentfernung bei Konsolidierung (Röntgen) nach ca. 2-3 Monaten. Keine routinemäßige postoperative Physiotherapie.

\section{Ergebnisse}

Im Zeitraum vom 01.01.1999 bis 31.12.2006 wurden insgesamt 85 Kinder mit einem Altersmedian von 6,1 Jahren wegen einer Condylus-radialis-humeri-Fraktur behandelt. 47 Patienten wurden operativ versorgt, davon 31 Kinder mit einer Kombination aus Schraube und Kirschner-Drähten, 13 Kinder mit einer Schraube allein und drei Kinder nur mit Kirschner-Drähten. Die Implantate wurden im Median 8,6 Wochen (5,0-17,1 Wochen) postoperativ entfernt. Insgesamt betrug die mediane Nachbeobachtungszeit 6 Monate (2-50 Monate). Bei keinem Patienten in dieser Serie traten Spätkomplikationen im Sinne einer nicht stattgefundenen Konsolidierung und/oder einer Pseudarthrosenbildung auf. In fünf Fällen mit Hypästhesien im Bereich der Haut über dem proximalen Radius kam es binnen 6 Monaten nach der Operation zu einer vollständigen Normalisierung. Im Rahmen eines Telefoninterviews im September 2007 konnten $87 \%$ der Patienten $(n=74)$ nachverfolgt werden. Drei Kinder gaben eine geringe Minderung der groben Kraft des verletzten Arms im Seitenvergleich an, wobei eines dieser Kinder zusätzlich ein geringes Ellenbogenflexionsdefizit berichtete, welches im Vergleich zur letzten klinischen Untersuchung nicht progredient war. In keinem der telefonisch kontaktierten Fälle war es nötig, den Patienten aufgrund persistierender Beschwerden und/oder eines subjektiv nicht akzeptablen Behandlungsergebnisses akut erneut einzubestellen.

\section{Schlüsselwörter}

Condylus-radialis-humeri-Fraktur - Pseudarthrose Ellenbogenluxation - Ellenbogenfraktur
Oper Orthop Traumatol 2010;22:81-91

DOI 10.1007/s00064-010-3006-2 


\section{Treatment of Lateral Humeral Condyle Fractures in Children}

\section{Abstract \\ Objective}

Surgical treatment of lateral humeral condyle fractures with reduction and retention in order to prevent lasting malalignment, pseudarthrosis, and joint instability.

\section{Indications}

Absolute: fractures with a complete dislocation or those in which plaster-free control X-ray on day 4 shows a gap of $>2 \mathrm{~mm}$.

Relative: complete fractures of the lateral humeral condyle which demonstrate a dislocation $\leq 2 \mathrm{~mm}$ on follow-up.

\section{Contraindications}

Incomplete, so-called hanging fractures of the lateral humeral condyle without notable secondary dislocation on follow-up.

\section{Surgical Technique}

Open reduction of the lateral humeral condyle via a lateral approach to the elbow joint. In smaller children $(<5$ years of age) fixation with Kirschner wires. In older children ( $\geq 5$ years of age) or in cases requiring compression radial screw fixation is recommended. In all cases, suture repair of the periosteum is advisable.

\section{Postoperative Management}

Long upper-arm plaster cast until wound healing is achieved. Subsequently, upper-arm plaster cast for 3-4 weeks postoperatively. Implants are removed following consolidation (confirmed by X-ray) after approximately 2-3 months. Routine physiotherapy is normally not required.

\section{Results}

From January 1, 1999 to December 31, 2006, 85 children with a median age of 6.1 years had lateral condyle fracture of the humerus treated. 47 patients underwent surgery. Of these, 31 were treated with a combination of screw and Kirschner wire fixation, 13 with a single screw, and in three cases, the fracture was fixed with Kirschner wires only. After a median of 8.6 weeks (range, 5.0-17.1 weeks), implants were removed. Median follow-up time was 6 months (range, 2-50 months). There were no late complications in this series (e.g., lack of consolidation, pseudarthrosis). In five cases, hyposensitivity of the skin above the proximal aspect of the radial bone was noted postoperatively. This problem was solved in all instances within the following 6 months. A telephone survey with a response rate of $87 \%$ (74 patients) was undertaken in September 2007. Three children noted a minimal deficiency in strength of the injured arm in comparison to the contralateral extremity. One of these children additionally stated a minor flexion deficit of the elbow already present at the last follow-up in the outpatient clinic, which showed no progress. None of the patients had to be referred back to the outpatient clinic because of persistent problems and/or unacceptable results.

\section{Key Words}

Lateral humeral condyle fractures · Pseudarthrosis . Elbow luxation · Elbow fracture

\section{Vorbemerkungen}

Die Fraktur des Condylus radialis humeri ist die häufigste Gelenkfraktur im Bereich der oberen Extremität im Wachstumsalter [12]. Ihr Anteil an allen Frakturen des distalen Humerus im Kindesalter beträgt 10-20\% [2]. Der Häufigkeitsgipfel dieser Fraktur liegt zwischen 4 und 8 Jahren.

Aufgrund der speziellen Anatomie sowie möglicher spezifischer Fehlbildungen weist diese Fraktur eine komplexe Problematik auf [9]. Der radiale Ellenbogenanteil steht, anders als der ulnare, aufgrund seiner anatomischen Situation unter speziellen Zug- und Druckbelastungen. Zum einen sind Radiusköpfchen und Capitulum humeri durch das in das Ligamentum anulare übergehende Seitenband fest miteinander verbunden. Zum anderen besteht aufgrund des physiologischen El- lenbogenvalgus eine größere Druckbelastung des radialen als des ulnaren Pfeilers. Da bei den meisten Frakturen des Condylus radialis humeri die Frakturfläche schräg von distoventral nach proximodorsal verläuft, ist durch den Druck des Radiusköpfchens, Fesselung des Seitenbands an das Ligamentum anulare und Ellenbogenvalgus eine zunehmende Dislokation des peripheren Fragments nach proximodorsal praktisch vorprogrammiert [10].

Von signifikanter Dislokation spricht man, wenn der Frakturspalt $>2 \mathrm{~mm}$ beträgt $[8,9]$. Bedingt durch die o.g. Problematik besteht die Gefahr, dass undislozierte Frakturen, auch im Rahmen einer Ruhigstellung im Gipsverband, sekundär dislozieren können.

Eine sekundäre Dislokation und die dadurch bedingte Unruhe in der Fraktur können zu einer Konsoli- 
dierungsverzögerung bis hin zur Pseudarthrose führen. Die Entwicklung einer Pseudarthrose ist allerdings nicht zwangsläufig gegeben. Auch nach sehr langer Konsolidierungszeit bis hin zu 2 Jahren kann die Fraktur noch knöchern ausheilen. Verbliebene Pseudarthrosen, die meist im Rahmen konservativ behandelter primär dislozierter Frakturen auftreten, führen in den meisten Fällen zu schwersten Gelenkdeformierungen. Durch das zunehmende Ausweichen des peripheren Fragments nach proximoradial kann es insbesondere zur zunehmenden Valgusfehlstellung mit der Gefahr einer späten Ulnarisläsion kommen [19]. Des Weiteren können, bedingt durch die radiale Instabilität, im Verlauf zunehmende Schmerzen auftreten. Häufig findet bei ausgeprägten Pseudarthrosen ein großer Teil der Ellenbogenflexion und -extension innerhalb der Pseudarthrose statt, so dass die Ellenbogenfunktion trotz der Gelenkdestruktion gut ist [9].

Es existieren unterschiedliche Einteilungen der Condylus-radialis-humeri-Fraktur. Milch [14] hat eine anatomische Klassifizierung dieser Frakturen vorgenommen:

- Typ-1-Fraktur: Die Frakturlinie verläuft durch den Epiphysenkern. Dieser Frakturtyp ist ausgesprochen selten.

- Typ-2-Fraktur: Die Frakturlinie beginnt in der posterolateralen Metaphyse, kreuzt die Epiphysenfuge mittig und verläuft in die Trochlea. Der Epiphysenkern wird nicht tangiert.

Beide Verletzungen entsprechen einer Salter-Harris-IV-Verletzung.

Die Klassifikation von Jakob et al. [6] orientiert sich am Dislokationsausmaß:

- Im Stadium I liegt eine undislozierte Fraktur vor, und die Gelenkfläche ist intakt. Es handelt sich somit um eine inkomplette, sog. hängende Fraktur, bei der der wesentliche Teil der Trochlea intakt bleibt.

- Im Stadium II liegt eine komplette Gelenkfraktur vor, die sekundär dislozieren kann.

- Stadium III stellt die komplett dislozierte Fraktur mit rotiertem Fragment dar (vgl. Abbildung 6a). Die Rotation des Fragments erfolgt durch die Extensorenmuskulatur des Unterarms.

Aufgrund des knorpeligen Gelenkanteils ist es oft schwierig, zwischen Stadium I und II zu differenzieren: Jakob et al. [6] haben in ihrer Arbeit aufzeigen können, dass es Frakturen gibt, die im knorpeligen Trochleaanteil noch „hängen“, es sich also um inkomplette, artikuläre Frakturen handelt, die nicht zur sekundären Dislokation führen und die, konservativ behandelt, folgenlos abheilen. Im Gegensatz dazu stehen die undislozierten, kompletten
Frakturen, die auch bei Ruhigstellung im Gipsverband zur sekundären Dislokation neigen [11]. Diese Frakturen gelten als instabil und müssen operativ behandelt werden, um einer späteren Konsolidierungsverzögerung und/oder Pseudarthrosenbildung vorzubeugen.

Grundsätzlich weisen Frakturen im Wachstumsalter überwiegend einen stereotypen Frakturverlauf auf. Dies gilt, wie Dallek \& Jungbluth [3] anhand der in das Capitulum humeri einstrahlenden Faserstruktur und des davon abhängigen initialen Frakturverlaufs nachweisen konnten, auch für die Frakturen des Condylus radialis humeri. Jedoch scheint der weitere Verlauf der Fraktur in den ulnaren Bereich hinein mehrere Varianten aufzuweisen: Die Fraktur kann hier einerseits im radiologisch nicht sichtbaren Teil der Trochlea bis ganz nach ulnar durch die gesamte Trochlea verlaufen. Andererseits kann sie auch als Kombination von fugenkreuzender, radialer Kondylenfraktur mit ulnarer Epiphysenlösung der Trochlea beobachtet werden. In der Literatur existieren keine Angaben über die prozentuale Verteilung dieser unterschiedlichen Frakturverläufe.

Thonell et al. [20] berichteten in ihrer Arbeit, dass $2,6 \%$ der radiologisch primär als stabil interpretierten Frakturen sekundär dislozieren. Die Dislokationsrate stieg bei Frakturen, die primär als nicht sicher stabil eingestuft wurden $(24 \%)$ bzw. als primär instabil galten (44\%), deutlich an. Bei Hasler \& von Laer [5] waren $16 \%$ der primär undislozierten Frakturen innerhalb der ersten 4 Tage trotz Ruhigstellung im Gipsverband sekundär disloziert. In einer weiteren Studie bei nicht oder nur gering dislozierten Frakturen lag der Anteil der Sekundärdislokation bei 10\% [16].

Die Standarddiagnostik ist eine radiologische Aufnahme des Ellenbogens in zwei Ebenen. Primär ist im anteroposterioren (a.p.) Bild oft nur ein kortikaler Unterbruch zu sehen. Beweisend ist stets im seitlichen Bild der von dorsal aus der Metaphyse nach ventral in die Epiphysenfuge verlaufende Frakturspalt [11].

Primär vollständig dislozierte Frakturen sind ohne Weiteres als solche zu erkennen und imponieren oft auch als dorsale Luxationsfrakturen des Ellenbogens. In letzterem Fall genügt in der Regel das Röntgen in einer einzigen Ebene, um die richtige Diagnose und die Weichen für die adäquate Therapie zu stellen. Bei den undislozierten Frakturen besteht die Schwierigkeit darin, dass radiologisch der knorpelige Anteil der Trochlea nicht beurteilt werden kann und so inkomplette, sog. hängende Frakturen von kompletten Frakturen nicht zu unterscheiden sind. In der Literatur wurden verschiedene Möglichkeiten vorgeschlagen, eine Unterscheidung o.g. Frakturtypen vorzunehmen $[1,7,15,22]$. Auf- 
grund einer unserer Meinung nach zu hohen Strahlenbelastung und Invasivität mit den damit verbundenen Schmerzen können wir die Durchführung einer Arthrographie des Ellenbogengelenks [13] oder gehaltene Aufnahmen [17] im Kindesalter nicht empfehlen.

Eine magnetresonanztomographische (MRT) Untersuchung ist eine sehr spezifische und nicht flächendeckend verfügbare, aufwendige Methode zur Unterscheidung einer inkompletten von einer kompletten Fraktur $[1,7,15]$. Ein weiterer Nachteil besteht darin, dass dieses Verfahren aufgrund der Dauer und des „Settings“ (starke Lärmbelästigung, enge Röhre) insbesondere bei jüngeren Kindern häufig eine Sedierung oder Narkose voraussetzt.

Eine elegante, kindgerechte, nichtinvasive Methode, die allerdings stark von der Erfahrung des Untersuchers abhängig ist, stellt die Ultraschalluntersuchung der Ellenbogenregion mittels Linearschallkopf dar [22].

\section{Operationsprinzip und -ziel}

Anatomische und sichere Refixation des abgerissenen Condylus radialis humeri. Behandlungsziel ist die exakte Wiederherstellung der Kongruenz der Gelenkflächen. Einerseits sollen damit der volle Bewegungsum-
Nach Durchsicht der Literatur $[5,10]$ ist davon auszugehen, dass komplett artikuläre, jedoch primär undislozierte Frakturen innerhalb der ersten 3-4 Tage nach dem Trauma auch bei Ruhigstellung im Gipsverband sekundär dislozieren. Aus diesem Grund empfehlen wir, bei allen nicht primär operativ behandelten Condylus-radialis-humeri-Frakturen ein gipsverbandfreies Röntgenbild (zwei Ebenen) um den 4. Tag nach Unfall durchzuführen. Als (sekundär) disloziert sind sämtliche Frakturen zu bezeichnen, die im zentralen Frakturbereich (a.p. Bild) eine Aufweitung des Frakturspalts $>2 \mathrm{~mm}$ aufweisen. In diesen Fällen kann sicher von einer Gelenkfraktur ausgegangen werden $[4,11]$, und die Indikation zur operativen Behandlung ist zu stellen.

fang des Ellenbogengelenks sowie die Gelenkstabilität mit einer sukzessiven kraftvollen, schmerzfreien Beweglichkeit wiederhergestellt werden. Andererseits gilt es, so eine sekundäre Wachstumsstörung mit konsekutiver Achsfehlstellung zu vermeiden.

\section{Relativ}

- Komplette Frakturen des Condylus radialis humeri, die im Verlauf eine Sekundärdislokation von $\leq 2 \mathrm{~mm}$ aufweisen.

\section{Kontraindikationen}

- Nicht dislozierte „hängende“ Fraktur ohne erkennbare Sekundärdislokation im Verlaufsröntgen nach 3-4 Tagen.

\section{Patientenaufklärung}

- Allgemeine Operationsrisiken (Infektion, Blutung).

- Iatrogene Läsion (selten!) des Nervus radialis oder seiner Hautäste durch direkte oder indirekte Manipulation (Dehnungsschaden).

- 4-wöchige postoperative Behandlung im Oberarmgipsverband (eine frühere Mobilisation mit funktioneller Nachbehandlung ist bei übungsstabiler Schraubenosteosynthese möglich).

- Klinische und radiologische Kontrolle nach 4 Wochen sowie nach 2-3 Monaten (vor Materialentfernung). 
- Pseudarthrosenbildung bei mangelnder Konsolidierung mit Valgusdeformität und möglicher späterer Irritation des Nervus ulnaris.

- Zunehmende Varisierung des Ellenbogengelenks durch Stimulation des radialen Fugenabschnitts.

- Notwendigkeit eines weiteren operativen Eingriffs in Narkose zur Materialentfernung.

\section{Operationsvorbereitungen}

- Beurteilung von peripherer Durchblutung, Motorik und Sensibilität: Prüfung und Dokumentation der Funktion der Armnerven, insbesondere der Nervi radialis et ulnaris.

- Schmerztherapie und Ruhigstellung im Rahmen der Erstversorgung: Analgosedierung in kindgerechter Dosierung (z.B. Midazolam-Hydrochlorid [Dormicum $^{\circledR}$ ] in Kombination mit Tramadol-Hydrochlorid [Tramal ${ }^{\circledR}$ ]) und Anlegen einer Oberarmschiene zur Ruhigstellung.

- Röntgenaufnahmen des Ellenbogens in zwei Ebenen. Falls die vorhandenen präoperativen Röntgenaufnahmen nicht konklusiv sind, evtl. Ultraschalluntersuchung oder Durchführung einer MRT-Untersuchung (ggf. in Sedierung/Kurznarkose).

\section{Instrumentarium und Implantate}

- Grundinstrumentarium.

- Bohrmaschine.

- Bohrdrahtspender (kurze Kirschner-Drähte) 160 mm (1,0 mm; 1,2 mm; 1,4 mm; 1,6 mm; 2,0 mm).

- Kleinfragmentzugschrauben oder kanülierte Schrauben mit selbstschneidendem Gewinde aus Titan (je 3,5-4,5 mm) mit entsprechender Unterlegscheibe.

\section{Anästhesie und Lagerung}

- Bevorzugt Allgemeinnarkose (Plexusanästhesie bei älteren Kindern/Jugendlichen alternativ möglich).

- Rückenlage.

- Seitentisch zur Armlagerung.

- Sterile Abdeckung, die ein Bewegen des Arms ermöglicht.

- Blutsperre am Oberarm.

- Antibiotikaprophylaxe bei geschlossenen Frakturen nicht standardisiert (Ausnahme: bekannter Herzfehler, fieberhafte bakterielle Infektion).

- Bildverstärker.

\section{Operationstechnik}

\section{Abbildungen 1 bis 5}

\begin{abstract}
Abbildung 1
Radialseitige, leicht geschwungene Hautinzision bei dezent gebeugtem Ellenbogengelenk. Die Inzision beginnt etwas proximal und nicht zu weit dorsal von dem in der Regel gut zu palpierenden Fragment.
\end{abstract}

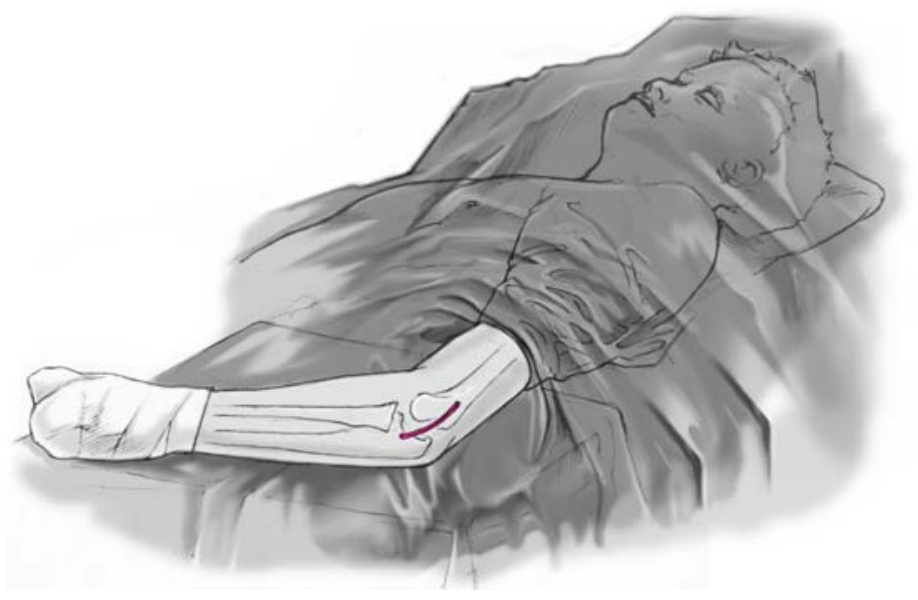


N. cutaneus antebrachii

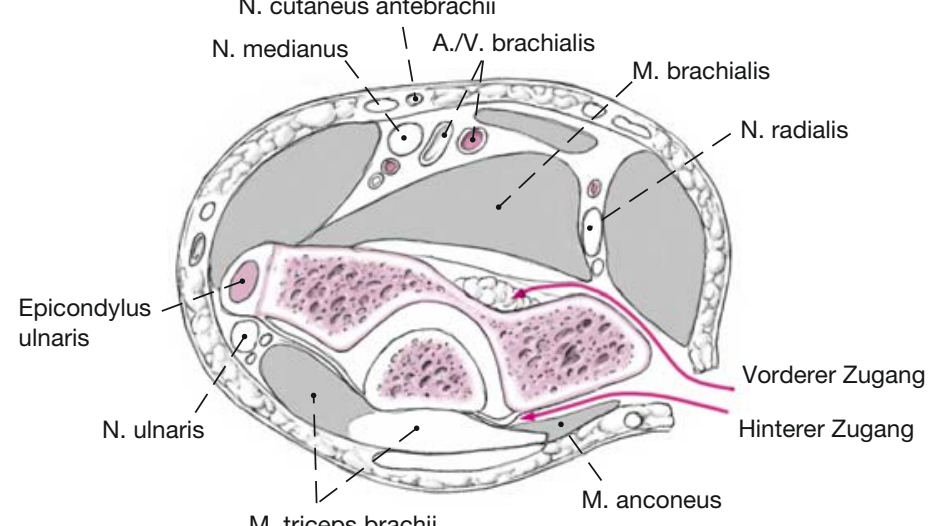

Fragment

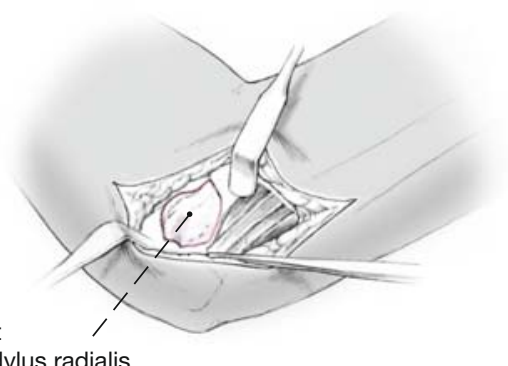

des Condylus radialis

\section{Gelenkknorpel}

des Condylus radialis

b



culus extensor carpi radialis brevis und Musculus extensor digitorum direkt auf die Gelenkkapsel (a). Sofern diese nicht schon zerissen ist, Eröffnung der Gelenkkapsel und Entlastung des Frakturhämatoms (b).

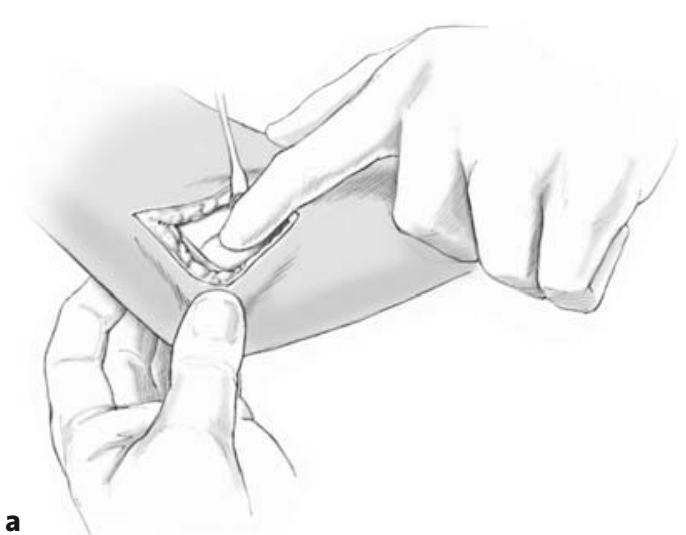

Abbildungen $3 a$ und $\mathbf{3} b$

Mehrmalige Spülung des Gelenks mit o,9\%iger NaCl-Lösung und Darstellung des Fragments. Unter Sicht wird das Fragment an die Trochlea angepasst und anatomisch reponiert (a). Das b

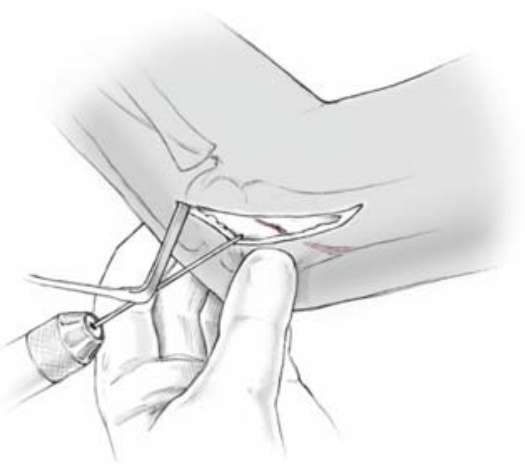

Repositionsergebnis wird entweder mit der Repositionszange oder mit einem von peripher distal-radial nach proximal-ulnar vorgeschobenen Kirschner-Draht gehalten (b). 


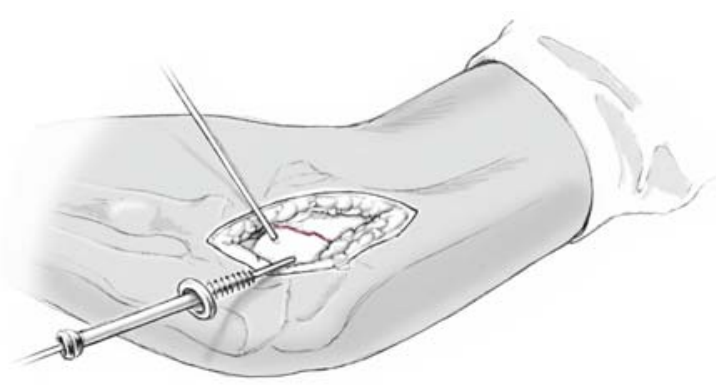

a

\section{Abbildungen 4a bis $4 c$}

Definitive Versorgung der Fraktur mit einer Kleinfragmentzugschraube oder mit einer selbstschneidenden kanülierten Titanschraube (Durchmesser je 3,5-4,5 mm mit Unterlegscheibe), welche von radial schräg in proximale Richtung eingebracht wird (Schonung der Wachsumsfuge, a). In Abhängigkeit von der Kompressionswirkung wird das Gewinde entweder in der Spongiosa des ulnaren Pfeilers oder (bei nicht ausreichender Festigkeit) in der metaphysären Gegenkortikalis verankert. Die Stabilität kann zusätzlich durch einen parallel zur Fuge in die Trochlea eingebrachten Kirschner-Draht vergrößert werden $(b, c)$.
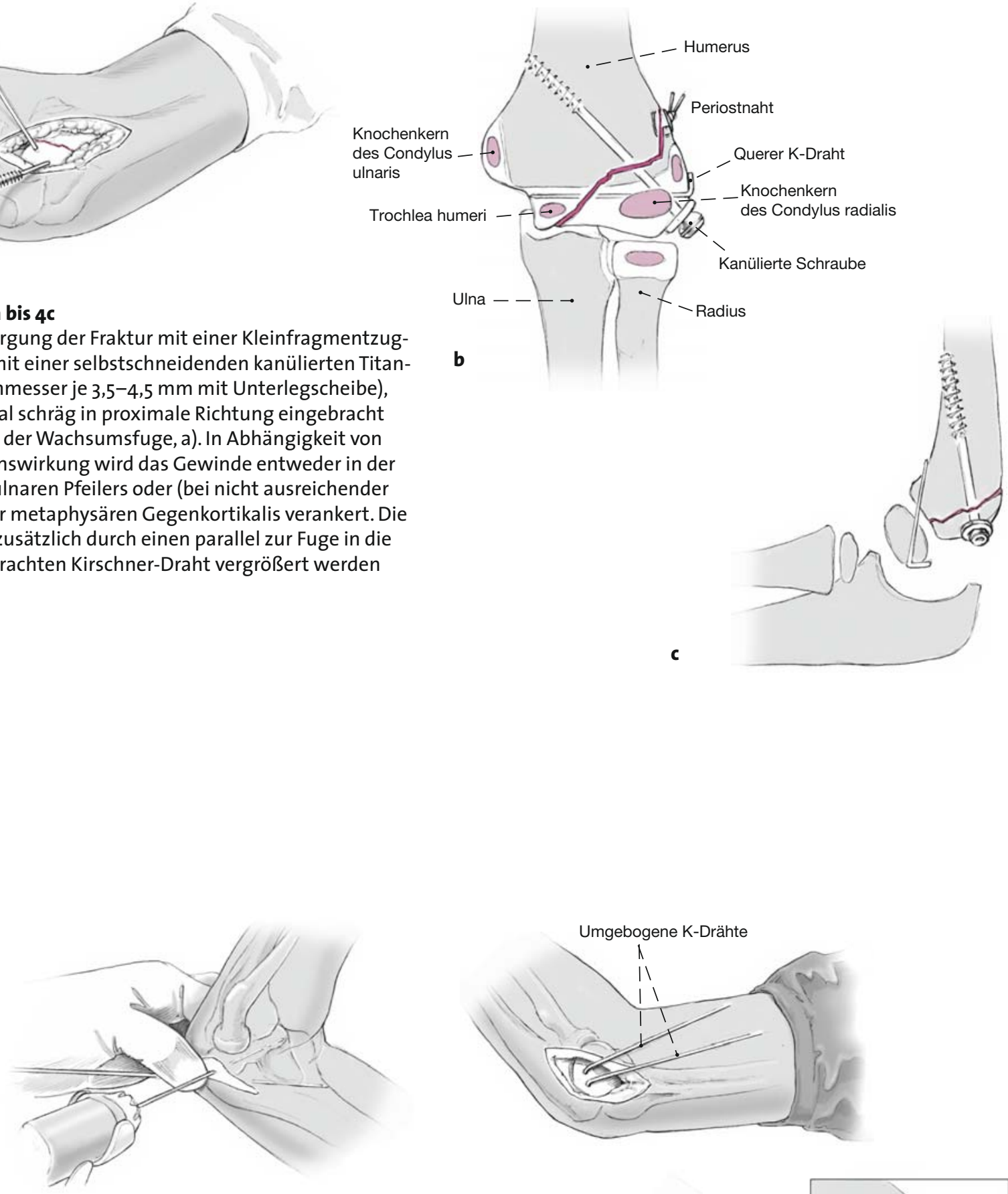

\section{Abbildung 5}

Alternativ, insbesondere bei kleineren Kindern ( $<5$ Jahre), kann die Stabilisation der Fraktur mit zwei Kirschner-Drähten erfolgen.

Zusätzlich sollte eine adaptierende radiodorsale Periostnaht erfolgen.






\section{Besonderheiten}

\section{Wahl des Implantats}

- Bei der Entscheidung, welches Implantat gewählt werden soll, sind neben dem Frakturtyp auch das Patientenalter und die Größe des Knochenfragments zu berücksichtigen. Ziel ist es, mit der jeweiligen Osteosynthese einerseits die Fraktur nach Reposition in einer anatomisch korrekten Position zu halten, andererseits eine ausreichende Kompression auszuüben, um einer späteren Pseudarthrose vorzubeugen.

- Tendenziell empfehlen wir bei kleineren Kindern (<5 Jahre) und einem eher kleinen Frakturfragment eine Kirschner-Draht-Osteosynthese (z.B. mit zwei Drähten à 1,6 mm). Bei älteren Kindern ( $\geq 5$ Jahre) und einem ausreichend großen Fragment bevorzugen wir die Schraubenosteosynthese, wobei die Schraube in Abhängigkeit von der Kompressionswirkung entweder in der Spongiosa des gegenüberliegenden ulnaren Pfeilers oder - bei nicht ausreichender Festigkeit - bis in die Gegenkortikalis eingebracht wird (vgl. Abbildung 6b). Gute Erfahrungen haben wir hierbei mit selbstschneidenden Titanhohlschrauben (3,5 oder 4,5 mm mit Unterlegscheibe) gemacht. Das Periost sollte, sofern möglich, zusätzlich mit einer Naht adaptiert werden, um die Stabilität zu erhöhen.

\section{Postoperative Behandlung}

- Mobilisation des Kindes ab dem Operationstag. Oberarmgipslonguette in $90^{\circ}$ Beugestellung im Ellenbogengelenk und mittlerer Rotation des Unterarms für 7-10 Tage. Der Arm kann dabei mit einer Armschlinge unterstützt werden. Verbandswechsel am 1. postoperativen Tag. Entlassung aus der stationären Behandlung bei komplikationslosem Verlauf ab dem 1. postoperativen Tag möglich. Auch eine tagesklinische Versorgung dieser Verletzung ist möglich. Bei Schulkindern ist der Schulbesuch ab dem 3. Tag nach der Entlassung gestattet.

- Nächster Verbandswechsel bei problemlosem Verlauf am 7.-10. postoperativen Tag mit Wundkontrolle und ggf. Nahtentfernung, sofern intraoperativ keine resorbierbare Hautnaht erfolgte. Im Anschluss Anlage eines geschlossenen Oberarmgipsverbands.

- Entfernung des Gipsverbands ca. 3-4 Wochen nach Operation in Abhängigkeit vom verwendeten Implantat (tendenziell ist eine längere Ruhigstellung bei alleiniger Kirschner-Draht-Osteosynthese notwendig) und gipsverbandfreies Konsolidierungsröntgen. Im Anschluss spontane Mobilisation des Ellenbogengelenks durch das Kind selbst abwarten. Eine Physio- oder Ergotherapie wird zu diesem Zeitpunkt nicht routinemäßig eingeleitet. Die Sportfähigkeit ist, eine schmerzfreie und ausreichende Beweglichkeit des Ellenbogengelenks vorausgesetzt, etwa 4-6 Wochen nach Frakturkonsolidierung gegeben.

- Implantatentfernung nach erneuter Röntgenkontrolle ca. 2-3 Monate nach der Erstoperation. Bei der Materialentfernung wird intraoperativ der passive Bewegungsumfang im Ellenbogengelenk geprüft und im Operationsbericht dokumentiert. Nach der Implantatentfernung wird das röntgenologische Ergebnis bei Bedarf dokumentiert.

- Im Verlauf vorsichtige, schmerzfreie Übungsbehandlung und geduldiges Abwarten der Selbstmobilisation des Patienten. Ein forciertes „Durchbewegen in Narkose" sollte hingegen im Kindesalter unterbleiben, da dies zu einer Verschlechterung der Bewegungseinschränkung und zur Ausbildung heterotoper Ossifikationen beitragen kann.

- Physiotherapeutische Übungsbehandlung vor allem bei älteren Kindern.

\section{Fehler, Gefahren, Komplikationen}

- Intraoperative Frakturierung des knöchernen Fragments durch Verwendung einer Repositionszange, bei wiederholten Bohrmanövern und Einbringen von zu dicken Kirschner-Drähten: In dieser Situation Fixieren jedes Fragments mit kleinen Kirschner-Drähten (z.B. 1,2 mm) und zusätzliche Adaptation mit resorbierbaren Nähten. Kleinere abgesprengte Fragmente können bei gleichzeitiger Refixation der größeren Fragmente entfernt werden.

- Kommt es beim Einsatz einer Schraubenosteosynthese zu einer Fraktur des Fragments, Fixation der einzelnen Fragmente, wie oben beschrieben, mit Kirschner-Drähten oder unter Verwendung einer Schraube mit einer „gezähnelten“ Unterlegscheibe.

- Bewegungseinschränkung im Ellenbogengelenk durch Refixation des Condylus radialis humeri in anatomisch nicht korrekter Position oder infolge von Vernarbungen der Gelenkkapsel und/oder von Bandstrukturen: Computertomographie (CT) des Ellenbogengelenks. Bei Bewegungseinschränkung ohne wesentliche knöcherne Veränderung Durchführung einer MRT. Sofern heterotope Ossifikationen nachgewiesen werden, ist insbesondere bei jüngeren Patienten eine abwartende Haltung angezeigt. In der Regel kommt es unter schmerzfreier Physiotherapie (ggf. Einnahme von nichtsteroidalen Antirheumatika) zu einem Remodeling und zu einer Verbesserung der Beweglichkeit.

- Selten kann es als Traumafolge zur avaskulären Nekrose des Capitulum humeri mit sekundärer Radius- 



Abbildungen $6 \mathrm{a}$ und $6 \mathrm{~b}$

a) Vollständig dislozierte Condylus-radialis-humeri-Fraktur mit rotiertem Fragment (Stadium III nach Jakob et al. [6]) bei einer 5-jährigen Patientin.

b) Konsolidierungsröntgen 7 Wochen nach offener Reposition und Fixation mittels einer kanülierten Zugschraube.

köpfchenluxation und ausgeprägter Valgusdefomität kommen [21]. Bildet sich in diesem Zusammenhang ein freies Fragment aus (Abklärung mittels CT), ist diese in Abhängigkeit von der Größe zu entfernen oder zu refixieren. Dieser Eingriff kann ggf. arthroskopisch erfolgen. Ohne Ausbildung eines freien Gelenkkörpers ist eine konservative Behandlung mit $\mathrm{Ru}$ higstellung über einige Wochen und längeren (Monate!) Verzicht auf belastende Sportarten (z.B. Tennis, Baseball, Kunstturnen) anzustreben. Oft kommt es im Verlauf trotz einer „Immobilisationsosteopenie“ zu einer Verbesserung der Hämodynamik und Regredienz der avaskulären Nekrose.

- Kosmetisch störende Varisierung der Ellenbogenachse, bedingt durch Konsolidierungsverzögerung mit Stimulation des radialen Fugenabschnitts: Sofern eine CT-Untersuchung in dieser Phase eine nicht oder nur wenig konsolidierte Fraktur zeigt, muss die Frakturstabilität hinterfragt werden. Gegebenenfalls besteht die Notwendigkeit zur Revisionsoperation mit Einbringen einer (neuen) Kompressionsschraube. Im Fall einer guten Konsolidierung und einer Achsabweichung von $<25^{\circ}$ empfehlen wir eine abwartende Haltung, da in der Regel keine signifikante Bewegungseinschränkung besteht. Bei Achsabweichungen $\geq 25^{\circ}$ tritt der „kosmetische Aspekt" in den Vordergrund. In solchen Fällen ist eine valgisierende, suprakondyläre Osteotomie nach Wachstumsabschluss zu diskutieren.
- Als Ausdruck einer zentralen Instabilität mit vorzeitigem Verschluss der Fuge und konsekutiver Einziehung der Trochlea kann sich im Verlauf eine sog. Fischschwanzdeformität ausbilden. Diese Komplikation imponiert im Wesentlichen radiologisch. Die Patienten sind klinisch kaum eingeschränkt, und die Deformität stellt an sich keine Präarthrose dar [18].

- Pseudarthrosenbildung und in der Folge mögliche Valgusdeformität der Ellenbogenachse mit Irritation des Nervus ulnaris als Spätkomplikation: Die Prognose und Art der Behandlung bei einer Pseudarthrose hängen u.a. von deren Lokalisation ab. Bei straffen und bei weit in den ulnaren Trochleabereich reichenden Pseudarthrosen kann die Gelenkfunktion durchKompression oder ggf. einfaches Anfrischen der Pseudarthrose mit anschließender Zugschraubenosteosynthese erhalten werden. Schwieriger ist die Situation bei Patienten, die über eine Pseudarthrose im Verlauf ein an der Ellenbogenfunktion beteiligtes Pseudogelenk (oft großes radiales Fragment) ausgebildet haben. Da hier eine Stabilisierung automatisch eine Funktionseinschränkung zur Folge hätte, empfehlen wir eher die suprakondyläre Osteotomie zur Korrektur der progredienten Valgusfehlstellung. Bei symptomatischer Instabilität mit Ulnarisirritation ist eine Korrektur mittels Osteosynthese, in therapierefraktären Fällen eine Ulnarisverlagerung indiziert [8]. Die Ergebnisse der operativen Behandlung von Pseudarthrosen des Condylus radialis humeri sind insgesamt als prognostisch unsicher anzusehen [23].

- Infektion: Gezielte systemische Antibiotikagabe und Ruhigstellung. Nur in schweren Fällen sind zusätzlich ein Wunddébridement mit Entfernung des Nahtmaterials und der Implantate sowie eine ausgiebige Gelenkspülung erforderlich. Gegebenenfalls Einlegen einer Gentamicin-Palacos-Minikette $\quad\left(\right.$ Septopal $^{\mathbb{Q}_{-}}$ Minikette, Biomet Merck GmbH, Berlin) für 5-7 Tage. Zur Entfernung des Antibiotikaträgers ist jedoch ein Zweiteingriff in Analgosedierung erforderlich.

\section{Ergebnisse}

Zwischen dem 01.01.1999 und dem 31.12.2006 wurden insgesamt 85 Kinder (59 Jungen, 26 Mädchen) mit einer 
Fraktur des Condylus radialis humeri behandelt. Die rechte Seite $(n=45)$ war etwas häufiger betroffen als die linke $(\mathrm{n}=40)$. Der Altersmedian zum Unfallzeitpunkt betrug 6,1 Jahre (2,6-12,5 Jahre). Eine operative Therapie erfolgte in $55 \%$ der Fälle $(n=47 ; 35$ Jungen, zwölf Mädchen). Die Zeit zwischen Unfall und operativer Versorgung betrug im Mittel 0,9 Tage (0-24 Tage). Die meisten Frakturen wurden mit einer Kombination aus Zugschraube und Kirschner-Draht versorgt $(\mathrm{n}=31)$. In 13 Fällen wurde die Fraktur lediglich mit einer Zugschraube (Abbildungen 6a und 6b) und bei drei Kindern ausschließlich mit Kirschner-Drähten stabilisiert.

Der Mittelwert des stationären Aufenthalts für alle Patienten $(\mathrm{n}=85)$ betrug 1,2 Tage ( $0-4$ Tage). Ein längerer stationärer Aufenthalt war insbesondere bei vorliegenden Begleitverletzungen erforderlich. Mit Ausnahme von Hypästhesien im Bereich der Haut am proximalen radialseitigen Unterarm bei fünf Patienten traten keine postoperativen Komplikationen auf.

Eine Oberarmgipsschiene wurde für durchschnittlich 3,7 Wochen (2,5-5 Wochen) angelegt. Alle Patienten wurden bis zur knöchernen Frakturkonsolidierung klinisch und radiologisch kontrolliert.

Die Implantate wurden im Median 8,6 Wochen (5,0-17,1 Wochen) postoperativ entfernt. Bei sechs Patienten war anschließend die Einleitung einer Physiotherapie wegen einer Bewegungseinschränkung im Ellenbogengelenk erforderlich. Insgesamt betrug die mediane Nachbeobachtungszeit 6 Monate (2-50 Monate). In keinen Fall traten Spätkomplikationen im Sinne einer nicht stattgefundenen Konsolidierung und/ oder einer Pseudarthrosenbildung auf. In den o.g. fünf Fällen mit Hypästhesien kam es binnen 6 Monaten nach der Operation zu einer vollständigen Normalisierung.

Im Rahmen eines Telefoninterviews (Tabelle 1) im September 2007 konnten wir 87\% $(n=74)$ der Patienten erreichen. Dadurch erhöhte sich die mediane Nachbeobachtungszeit auf 66 Monate (2-101 Monate). Erhoben wurden Informationen bezüglich der früheren und möglichen aktuellen Beschwerden, Kraftminderung des betroffenen Unterarms und der Hand sowie offensichtlicher Bewegungseinschränkungen des verletzten Ellenbogens im Vergleich zur unverletzten Gegenseite (s. Tabelle 1). Aufgrund des Interviews wurde eine Einschätzung der klinischen Ergebnisse als gut, akzeptabel und nicht akzeptabel vorgenommen. Der Großteil der befragten Patienten $(\mathrm{n}=71$ [96\%]) wies ein gutes Ergebnis mit keinerlei subjektiv empfundenen Einschränkungen auf. Drei Fälle wurden als ,akzeptabel“ kategorisiert, da eine geringe Minderung der groben Kraft des verletzten Arms im Seitenvergleich beschrieben wurde.

\section{Tabelle 1}

Standardisierte Fragen im Telefoninterview.

1. Bist du (ist Ihr/e Tochter/Sohn) zurzeit beschwerdefrei? (Differenzierung in: a) ja, b) nein)

2. Hast du (hat Ihr/e Tochter/Sohn) eine Kraftminderung des betroffenen Unterarms und der Hand? (Differenzierung in: a) keine, b) geringe [Patient nimmt Kraftminderung wahr, wird aber im Alltag, z.B. Sport, Schule, Hobby, Ausbildung, dadurch nicht behindert], c) signifikante [Kraftminderung wirkt sich auf Alltagsaktivität aus])

3. Hast du (hat Ihr/e Tochter/Sohn) Bewegungseinschränkungen des verletzten Ellenbogens für Beugung/Streckung (Flexion/Extension) oder Drehung (Pronation/Supination) im Vergleich zur Gegenseite? (Differenzierung in: a) keine, b) geringe [Patient nimmt Defizit wahr, wird aber im Alltag, z.B. Sport, Schule, Hobby, Ausbildung, nicht eingeschränkt], c) signifikante [Bewegungseinschränkung wirkt sich auf Alltagsaktivität aus])

Eines dieser Kinder gab zudem ein geringes Ellenbogenflexionsdefizit im Vergleich zur Gegenseite an. In keinem der telefonisch nachuntersuchten Fälle war es nötig, den Patienten aufgrund persistierender Beschwerden und/oder eines nicht akzeptablen Behandlungsergebnisses persönlich erneut einzubestellen.

\section{Literatur}

1. Betran J, Rosenberg ZS, Kawelblum M, et al. Pediatric elbow fractures: MRI evaluation. Skeletal Radiol 1994;23:277-81.

2. Chambers HG, Wilkins KE. Fractures and dislocations of the elbow region. In: Rookwood CA Jr, Wilkins KE, Beaty JH, eds. Fractures in children, 5th edn. Philadelphia: Lippincott-Raven, 2001:653-69.

3. Dallek $\mathrm{M}$, Jungbluth $\mathrm{KH}$. Histomorphologische Untersuchungen zur Entstehung der Condylus-radialis-humeri-Fraktur im Wachstumsalter. Unfallchirurgie 1990;16:57-62.

4. Foster DE, Sullivan JA, Gross RH. Lateral humeral condylar fractures in children.J Pediatr Orthop 1985;5:16-22.

5. Hasler C, von Laer L. Schraubenosteosynthese bei dislozierten Frakturen des Condylus radialis humeri im Wachstumsalter - eine prospektive Langzeitstudie. Unfallchirurgie 1998;101:280-6.

6. Jakob R, Fowles JV, Rang M, et al. Observations concerning fractures of the lateral humeral condyle in children. J Bone Joint Surg Br 1975;57:430-6.

7. Kamegaya $M$, Shinohara $Y$, Kurokawa $M$, et al. Assessment of stability in children's minimally displaced lateral humeral condyle fracture by magnetic resonance imaging. J Pediatr Orthop 1999;19:570-2.

8. Kiderlen MJ, Schlickewei W. Operationsverfahren bei intraartikulären distalen Humerusfrakturen im Wachstumsalter. Oper Orthop Traumatol 2008;20:423-34.

9. von Laer L. Fracture of condylus radialis humeri during skeletal growth. Arch Orthop Trauma Surg 1981;98:275-83.

10. von Laer L. Die Fraktur des Condylus radialis humeri im Wachstumsalter. Unfallchirurg 1998;101:271-9.

11. von Laer L. Frakturen und Luxationen im Wachstumsalter, 5. Aufl. Stuttgart: Thieme, 2007:153-70.

12. Landin LA, Danielsson LG. Elbow fractures in children. An epidemiological analysis of 589 cases. Acta Orthop Scand 1986;57:309-12.

13. Marzo JM, d'Amato C, Strong M, et al. Usefulness and accuracy of arthrography in management of lateral humeral condyle fractures in children. J Pediatr Orthop 1990;10:317-21.

14. Milch HE. Fractures and fracture-dislocations of the humeral condyles. J Trauma 1964;15:592-607. 
15. Nimkin K, Kleinman PK, Teeger S, et al. Distal humeral physeal injuries in child abuse: MR imaging and ultrasonography findings. Pediatr Radiol 1995;25:562-5.

16. Pirker ME, Weinberg AM, Höllwarth ME, et al. Subsequent displacement of initially nondisplaced and minimally displaced fractures of the lateral humeral condyle in children. J Trauma 2005;58:1202-7.

17. Rang M, Thompson GH. Children's fractures: principles and management. Reconstr Surg Traumatol 1979;17:2-15.

18. Rutherford A. Fractures of the lateral humeral condyle in children. J Bone Joint Surg Am 1985;67:851-6.

19. Tarczyńska M, Kołodziej R, Gagała J. Results of operative treatment for ulnar neuropathy in patients with a history of fracture of the lateral part of the humeral condyle in childhood. Ortop Traumatol Rehabil 2007;9:75-81.

20. Thonell S, Mortensson W, Thomasson B. Prediction of the stability of minimally displaced fractures of the lateral humeral condyle. Acta Radiol 1988;29:367-70.

21. Vocke-Hell AK, von Laer L, Slongo T, et al. Secondary radial head dislocation and dysplasia of the lateral condyle after elbow trauma in children. J Pediatr Orthop 2001;21:319-23.
22. Vocke-Hell AK, Schmid A. Sonographic differentiation of stable and unstable lateral condyle fractures of the humerus in children. J Pediatr Orthop B 2001;10:138-41.

23. Wilkins KE. Residuals of elbow trauma in children. Orthop Clin North Am 1990;21:291-314.

\section{Korrespondenzanschrift}

Dr. Rainer Kubiak

Abteilung für Traumatologie

Chirurgische Klinik

Universitäts-Kinderspital

Steinwiesstraße 75

8032 Zürich

Schweiz

Telefon (+41/44) 2667-634, Fax -170

E-Mail: rainerkubiak@hotmail.com

\section{OOT im Internet - Suche nach Autoren und Stichworten}

Die Zeitschrift „Operative Orthopädie und Traumatologie" ist seit mehreren Jahren auch im Internet mit einer eigenen Homepage präsent. Auf dieser Seite können Sie nach bereits erschienenen Beiträgen suchen, allgemeine Informationen finden, Schriftleitung und Redaktion kontaktieren und vieles mehr. Sie finden uns unter www.oot.at. Für die Suche nach Autoren und Stichworten geben Sie einfach einen Begriff in das Suchfenster ein. Sie können Ihre Suche im Anschluss bei Bedarf noch auf einen einzelnen Jahrgang begrenzen. 